\title{
A case of Meckel's diverticulum with life-threatening recurrent gastrointestinal bleeding in an adult
}

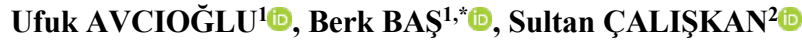 \\ ${ }^{1}$ Department of Gastroenterology, Faculty of Medicine, Ondokuz Mayıs University, Samsun, Turkey \\ ${ }^{2}$ Department of Patology, Faculty of Medicine, Ondokuz Mayıs University, Samsun, Turkey
}

\begin{abstract}
\begin{tabular}{ccccc}
\hline Received: 09.09 .2020 & $\bullet$ & Accepted/Published Online: 10.11 .2020 & Final Version: 18.03 .2021 \\
\hline
\end{tabular}
\section{Abstract}

Meckel's diverticulum is the most common congenital anomaly of the gastrointestinal system. It results from the incomplete closure of the omphalomesenteric canal in intrauterine life. It is mostly diagnosed in childhood with intestinal obstruction and bleeding. It is uncommon and often clinically silent in adults. Also, it is difficult to diagnose the patients with symptomatic Meckel's diverticulum. We report a 28-year-old male who presented with life-threatening recurrent lower gastrointestinal (GI) bleeding and was operated with the diagnosis of Meckel's diverticulum with duodenal ulcer.
\end{abstract}

Keywords: congenital anomaly, Meckel's diverticulum, recurrent gastrointestinal bleeding

\section{Introduction}

Meckel's diverticulum is the most common congenital anomaly of the gastrointestinal tract (Sagar et al., 2006). It results from the incomplete closure of the omphalomesenteric canal in intrauterine life. The incidence in the general population has been estimated to be about 2 percent (Lüdtke et al., 1989). It occurs more frequently in men than in women. It is a true diverticulum so it contains all normal layers of the intestinal wall and results from the incomplete closure of the omphalomesenteric canal (DeBartolo and Van Heerden, 1976). It is mostly diagnosed in childhood with intestinal obstruction and bleeding. It is uncommon and often clinically silent in adults. Also, it is difficult to diagnose the patients with symptomatic Meckel's diverticulum. We report a 28-year-old male who presented with life-threatening recurrent lower gastrointestinal (GI) bleeding and was operated with the diagnosis of Meckel's diverticulum with duodenal ulcer.

\section{Case report}

A 28-year-old male patient, with no medical or surgical history, presented to the emergency department with complaint of hematochezia for three days. According to the history of the patient, the bleeding started suddenly three days ago and gradually increased. He had bright red, jelly-like stool with an amount of approximately $200 \mathrm{ml}$, four or three times in the last 24 hours. He had also fatigue, dizziness and cold sweating. On physical examination, the patient was afebrile $\left(36.4^{\circ} \mathrm{C}\right)$ with a pulse rate of 105 beats per minute, respiratory rate of 22 breaths per minute, blood pressure of $130 / 70 \mathrm{~mm} \mathrm{Hg}$ on lying and
$110 / 60 \mathrm{mmHg}$ on standing. The sclera of both eyes was pale, abdominal examination was normal and bright red bloody contamination was observed on rectal examination. The patient's reference laboratory values were as follows: Hemoglobin: 7.4 g/dl, Platelet: 177.000/Ul, Hematocrit: 33.7\%, MCV: 72fL, Wbc: 8300 / Ul, Creatine: $1.31 \mathrm{mg} / \mathrm{dl}$, AST: 26 U / 1, ALT: 14 U / 1, Na: $141 \mathrm{mEq} / \mathrm{L}, \mathrm{K}: 4.2 \mathrm{mEq} /$ L, Ca: $9.6 \mathrm{mg} / \mathrm{dl}$ and INR: 1.2 .

During the follow-up of the patient, two more abundant bloody stools occurred and one unit of erythrocyte suspension was given. Upper GI endoscopy and colonoscopy examination was performed. In the upper GI endoscopy of the patient, there was no remaining or fresh blood in the stomach, Forest $2 \mathrm{c}$ ulcer was observed in the bulb and was intervened with Argon plasma coagulation. On colonoscopy, fresh blood was seen in the terminal ileum and all colon segments. No active bleeding focus was observed.

Since it was thought that the current clinical status of the patient could not be explained by the ulcer in the bulb, CT angiography was performed to detect the bleeding focus. At the umbilical level, posterior to the distal ileal segment, a tubular structure with mucosal thickening, intraluminal air, and blind ending (approximately $45 \mathrm{~mm}$ in length and $15 \mathrm{~mm}$ in thickness) was seen and interpreted as Meckel's diverticulum (Figure 1). Since the bleeding continued during follow-up, the patient was consulted to the general surgery department, and partial small bowel resection and end-to-end anastomosis were 
performed. The pathology of the small intestine resection material was compatible with Meckel's diverticulum containing ectopic gastric mucosa (Figure 2).

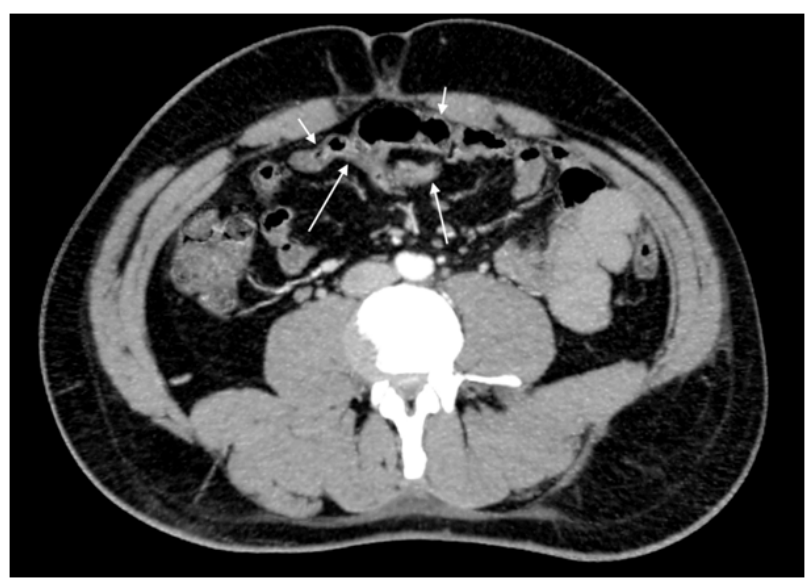

Fig. 1. Meckel's diverticulum seen at the umbilical level on CT angiography

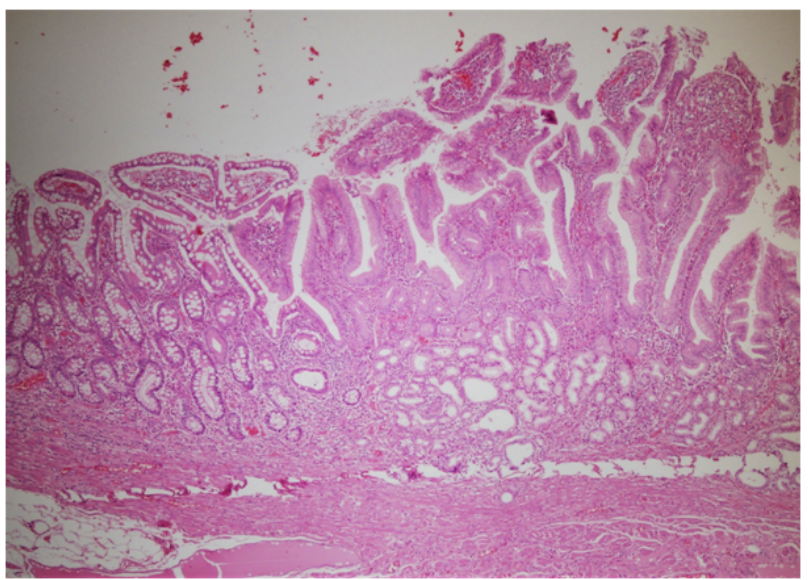

Fig. 2. Normal small intestinal mucosa (left side) followed by gastric mucosa (right side) (H\&E stain, x400 magnification)

\section{Discussion}

Meckel's diverticulum is often clinically silent in adults compared to children, but complications are more serious. The most common clinical finding is gastrointestinal system bleeding, as in our patient (Park et al., 2005). In addition to bleeding, it may also present with other clinical findings such as obstruction, invagination, diverticulitis, and perforation. It usually contains intestinal mucosa, but may contain gastric, colon, duodenal and pancreatic tissue. As in our patient, bleeding in Meckel's diverticulum containing gastric tissue usually develops on the basis of peptic ulcers due to acid secreted from the ectopic gastric mucosa. Bleeding due to Meckel's diverticulum may be overt or occult (Slívová et al., 2018). Some risk factors have been defined for Meckel's diverticulum to be symptomatic. These risk factors are; age under 50, male gender, diverticulum larger than $2 \mathrm{~cm}$ and those contained histologically abnormal tissue. All of these risk factors were present in our patient (Park et al., 2005).

There are no specific laboratory and physical examination findings for the bleeding due to Meckel's diverticulum. In the approach to bleeding, the priority is endoscopic evaluation likewise for all gastrointestinal system bleeding. In patients presenting with bleeding due to Meckel's diverticulum, there is usually no pathology in endoscopic evaluation. Because of this, other imaging methods such as CT Angiography and scintigraphy are referred (Enge and Frimann-Dahl, 1964). Duodenal ulcer was detected in our patient, which could explain the bleeding. However, further examination was required because the patient had hematemesis rather than melena, fresh blood was seen in the terminal ileum in colonoscopy, and the duodenal ulcer did not bleed during the endoscopic procedure.

CT angiography is an important diagnostic method to show low amount of bleeding up to $0.3 \mathrm{ml} / \mathrm{min}$, but it is not preferred in special patient groups such as pregnant women, kidney failure patients and those who have contrast allergy due to the need for iv contrast medium. Scintigraphy is based on the principle of the uptake of Tc-99m pertechnetate by the gastric mucosa (Lin et al., 2002). It has 51-92\% positive results in lower gastrointestinal system bleeding and can detect bleeding between $0.1-0.3 \mathrm{ml} / \mathrm{min}$. The positive predictive value of scintigraphy in adults is low $(60 \%)$. There are promising studies on other methods, especially double balloon enteroscopy. Retrospective studies involving large populations have demonstrated that double balloon enteroscopy has higher diagnostic accuracy than scintigraphy. In addition, it allows treatment in some cases although it is limited (Geng et al., 2017). However, its disadvantages are that it is technically difficult, it is not available in most endoscopy centers, and optimal imaging cannot be obtained due to blood and bleeding residues accumulated in the lumen during bleeding.

In patients presenting with bleeding due to Meckel's diverticulum, the first treatment approach is to start proton pump inhibitors. Proton pump inhibitors do not reduce the diagnostic sensitivity of scintigraphy. Our patient continued to bleed under PPI treatment because of the duodenal ulcer (Bandi et al., 2017). Surgery is the next treatment approach for persistent gastrointestinal system bleeding despite proton pump inhibitor therapy. Surgical options are diverticulectomy or segmental resection (Clark et al., 2008). Segmental resection is generally preferred in lesions larger than $2 \mathrm{~cm}$, as in our patient (Hosn et al., 2014).

In conclusion, Meckel's diverticulum is a rare condition in the adult population. However, it should be kept in mind as a differential diagnosis for every patient presenting with lower GI bleeding and whose bleeding etiology cannot be detected in endoscopic examination, so additional radiological and scintigraphic examinations should be performed for diagnosis.

\section{Conflict of interest}

All authors declare no conflict of interest regarding this manuscript.

\section{Acknowledgments}

No competing financial interests exist. 


\section{References}

1. Bandi, A., Tan, Y.W., Tsang, T., 2014. Correlation of gastric heterotopia and Meckel's diverticular bleeding in children: A unique association. Pediatr. Surg. Int. 30 (3), 313-316.

2. Clark, J.M., Koontz, C.S., Smith, L.A., et al., 2008 Video-assisted transumbilical Meckel's diverticulectomy in children. Am. Surg.74(4), 327-329.

3. DeBartolo, H.M., Jr, van Heerden J.A., 1976. Meckel's diverticulum. Ann. Surg. 183(1), 30-33.

4. Enge, I., Frimann-Dahl, J., 1964. Radiology in acute abdominal disorders due to meckel's divertıculum. Br. J. Radiol. 37, 775-780.

5. Geng, L.L., Chen, P.Y., Wu, Q., et al., 2017. Bleeding Meckel's Diverticulum in Children: The Diagnostic Value of DoubleBalloon Enteroscopy. Gastroenterol. Res. Pract. 2017, 7940851.

6. Hosn, M.A., Lakis, M., Faraj, W., Khoury, G., Diba, S., 2014. Laparoscopic approach to symptomatic meckel diverticulum in adults. JSLS. 18(4), e2014.00349.
7. Lin, S., Suhocki, P.V., Ludwig, K.A., Shetzline, M.A., 2002. Gastrointestinal bleeding in adult patients with Meckel's diverticulum: the role of technetium $99 \mathrm{~m}$ pertechnetate scan. South Med. J. 95(11), 1338-1341.

8. Lüdtke, F.E., Mende, V., Köhler, H., Lepsien, G., 1989. Incidence and frequency or complications and management of Meckel's diverticulum. Surg. Gynecol. Obstet. 169(6), 537-42.

9. Park, J.J., Wolff, B.G., Tollefson, M.K., Walsh, E.E., Larson, D.R., 2005. Meckel diverticulum: The Mayo Clinic experience with 1476 patients (1950-2002). Ann. Surg. 241(3), 529-533.

10. Sagar, J., Kumar, V., Shah, D.K., 2006. Meckel's diverticulum: a systematic review [published correction appears in J. R. Soc. Med. 99(10), 501-505.

11. Slívová, I., Vávrová, Z., Tomášková, H., Okantey, O., Penka, I., Ihnát, P., 2018. Meckel's Diverticulum in Children-Parameters Predicting the Presence of Gastric Heterotopia. World. J. Surg. 42(11), 3779-3784. 